\title{
Highly engineered mesoporous structures for optical processing
}

\author{
By G. J. Parker ${ }^{1, *}$, M. D. B. Charlton ${ }^{1,2}$, M. E. Zoorob ${ }^{2}$, \\ J. J. BaumberG ${ }^{1,2,3}$, M. C. Nettit $^{2}$ and T. LeE ${ }^{1}$ \\ ${ }^{1}$ School of Electronics and Computer Science, The University of Southampton, \\ Southampton SO17 1BJ, UK \\ ${ }^{2}$ Mesophotonics Ltd, Southampton SO16 7NP, UK \\ ${ }^{3}$ School of Physics, The University of Southampton, Southampton SO17 1BJ, UK
}

Arranging periodic, or quasi-periodic, regions of differing refractive index in one, two, or three dimensions can form a unique class of mesoporous structures. These structures are generally known as photonic crystals, or photonic quasicrystals, and they are the optical analogue of semiconducting materials. Whereas a semiconductor's band structure arises from the interaction of electron or hole waves with an arrangement of ion cores, the photonic crystal band structure results from the interaction of light waves with an arrangement of regions of differing refractive index.

What makes photonic crystals highly attractive to the optical engineer is that we can actually place the regions of differing refractive index in a pattern specifically tailored to produce a given optical function, such as an extremely high dispersion, for example. That is, we can define the geometrical arrangement of the dielectric foam to provide us with the form of band structure we require for our optical functionality.

In this paper, the optical properties and applications of these highly engineered mesoporous dielectrics will be discussed.

Keywords: photonic crystals; superprisms; multirefringence; photonic quasicrystals; planar optical waveguides; pinwheel construction

\section{Porous structures for optical processing (photonic crystals)}

The porous structures to be described require refractive index variations on a length-scale commensurate with the wavelength of light. Such structures are usually called photonic crystals (PCs) first discussed by Bykov (1972), and the refractive index variation is often achieved, in planar devices, by etching air holes into dielectric waveguiding materials. To be more precise, the length-scales we consider are of the order of the light wavelength divided by the effective index of the matrix material propagating the light. Hence, for a PC device operating in the visible part of the spectrum, the length-scale of the refractive index variation is in the region of a few hundred nanometres. Lying between the

* Author for correspondence (gjp@ecs.soton.ac.uk).

One contribution of 18 to a Discussion Meeting Issue 'Engineered foams and porous materials'. 


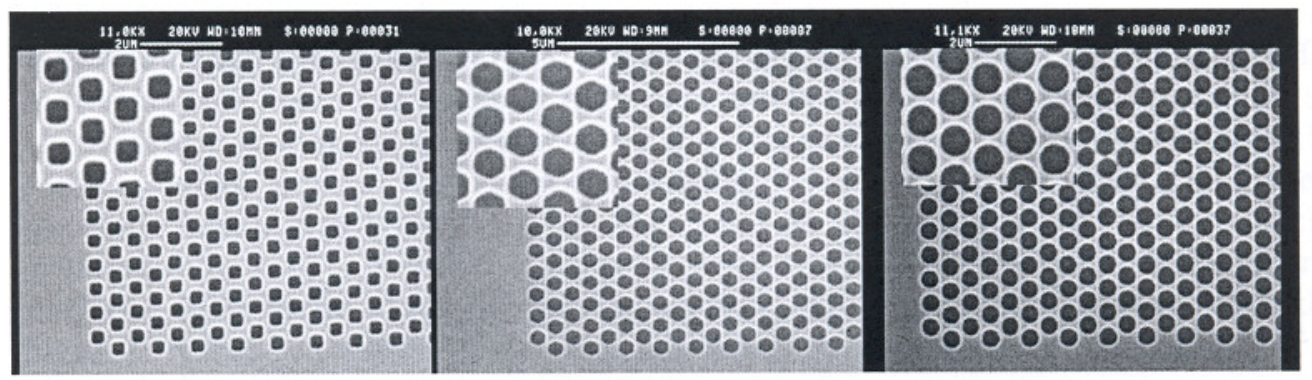

Figure 1. E-beam-defined submicron pores in silicon nitride.

micrometre $\left(10^{-6} \mathrm{~m}\right)$ and nanometre $\left(10^{-9} \mathrm{~m}\right)$ regimes, I will use the term mesoporous to describe these porous materials.

Figure 1 shows a plan-view scanning electron microscope (SEM) image of triangular lattice of air holes dry-etched into a silicon nitride waveguiding core layer. A further magnified region of the PC structure is shown in the top lefthand corner of each image. Each etched 'hole' is individually written using a direct write electron beam lithography machine, and it can be seen that the 'holes' are square-shaped in the first image, hexagonal in the second and round in the third. We thus have complete control over both the basis and the lattice of the photonic 'crystal', and, therefore, together with the choice of material, we have considerable flexibility in the overall design of these structures.

In order to understand how these PC devices operate in the optical region, it is fortunate that we can form direct analogies with the more familiar case of charge carrier propagation in semi-conducting materials. The interaction of the electron (or hole) wave with the periodic electrostatic potential provided by the lattice of ion cores in a semi-conducting crystal gives rise to allowed and forbidden bands of conduction. The electronic band gap is an energy region where no carrier propagation is allowed, in any direction, and if we draw a picture of the allowed regions as a function of electron energy and momentum (an $E-k$ diagram), we obtain a rich band structure that provides us with many interesting properties and opportunities. The situation is precisely the same for PCs provided that we make the following link: the periodic variation in refractive index caused by etching air holes in a dielectric, looks to the propagating photons, just like the periodic variation in potential does to charge carriers in a semiconductor. Variations in refractive index in $\mathrm{PCs}$ are, therefore, analogous to variations in electrostatic potential in semiconductors, and in both cases we have the interaction of a wave (whether photon, electron or hole) with a periodic 'potential'. Since we are dealing with very similar physical situations, the equations describing these systems will be similar, with similar solutions. We should not draw too close an analogy here because in the electronic case we are dealing with solutions to the scalar Schrödinger equation, and in the PC case we solve the vector Maxwell equations, so we can expect differences in detail between these two systems. Fortunately, the gross properties of the two systems do not differ significantly, in the analogy, so we can form useful mental images of the processes in the PC case given our basic understanding of solid-state electronics.

The magnitude of the band gap in the electronic case is defined by the energy difference that occurs for carriers either in the region of the ion cores, or at 
midway positions, i.e. where the electrostatic interaction is either a maximum or minimum with the charge carrier. Hence, a bigger (wider) band gap will occur for materials that have the greatest difference between the maximum and minimum electrostatic potentials experienced by the charge carriers in moving through the semiconductor crystal. Carrying this analogy over into the PC case, the largest photonic band gaps will occur (if they occur at all) in materials that have the greatest difference in refractive index between the air hole regions and the matrix material (dielectric). This is why the earliest experimentalists worked with the highest refractive index dielectrics in order to increase the likelihood of a photonic band gap opening up.

There is one more point to consider before moving onto the fabrication and practical applications of PCs, and that is the role of the structure. Even with a large refractive index contrast an optical band gap still may not form if an inappropriate lattice is chosen, so the geometry itself becomes very important. Again, even a simple mental image can prove useful in understanding this. Consider a simple cubic arrangement of high index spheres. Then it is clear that there are a few 'preferred' directions where the lattice periodicity of the correct length-scale occurs, and many directions where index variations over the required length-scale do not occur. It therefore seems quite likely that in a simple cubic lattice structure (or square lattice in a planar device) that we will be able to find directions where Bragg reflection will not occur, and, hence, in these directions there will be no apparent band gap. In these cases, we say the band gap is incomplete. Our chances of creating a complete band gap (all directions and all polarization states) will be increased if we are able to 'fill in the gaps' in our simple cubic structure. We can do this of course by moving to higher orders of lattice symmetry such as that provided by a face-centred cubic (FCC) or diamond structure. It is precisely for these reasons that Yablonovitch (1987) chose the material and lattice structure he did when creating the first PC operating in the microwave region of the electromagnetic spectrum. Why the microwave region? Because the fabrication process Yablonovitch used involved the mechanical drilling of an FCC lattice of air holes into a dielectric block (subsequently this material was called Yablonovite) and physically this meant that the length-scale, or periodicity of the structure, would be in the millimetre regime. In order to move the band gap into the visible region we need to use fabrication techniques utilized by the semiconductor industry, to obtain porous materials with refractive index variations in the range of a few hundred nanometres - and even then it is still a very difficult technology to implement effectively!

At the University of Southampton we started by following the most logical route; i.e. we chose a matrix material with a high refractive index that was compatible with fabrication in a semiconductor clean room environment. Fortunately, that high index matrix material was silicon $(n \sim 3.6)$, so that the fabrication processes we needed to use were already to hand. We decided not to be too ambitious in the first instance and so worked with two-dimensional structures (planar devices) rather than trying to fabricate full three-dimensional devices. The technique we used to etch these silicon structures was first described by Lehmann (1993) and it is a photoelectrochemical etching process.

Figure 2 shows the starting point for producing macroporous silicon structures for optical applications. This is an SEM of a (100) silicon surface that has been 


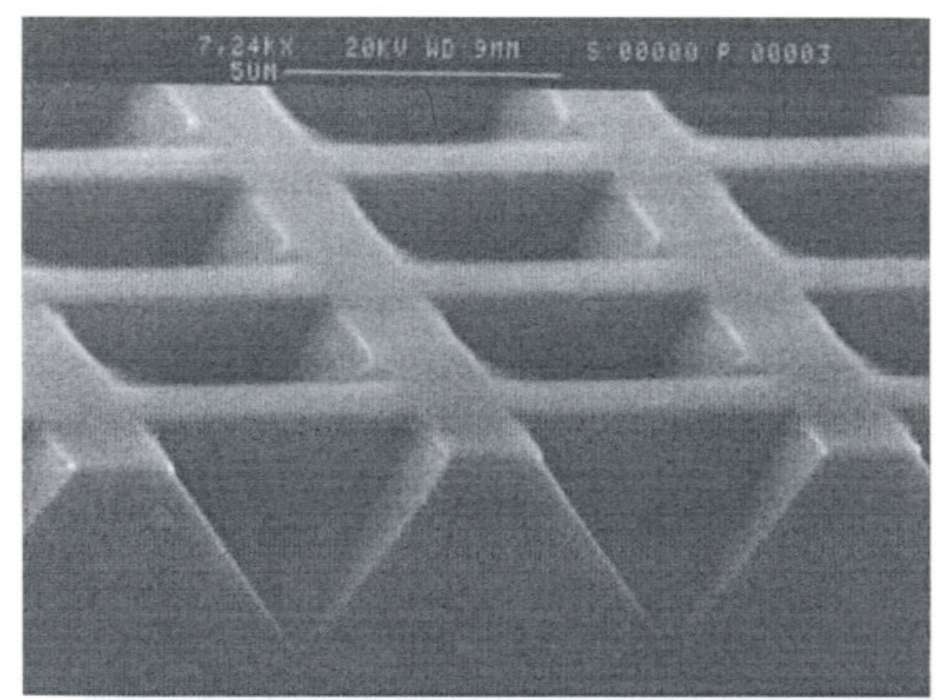

Figure 2. KOH-etched inverted pyramids in silicon.

lithographically patterned and etched using potassium hydroxide (KOH etch). This wet etch preferentially etches along certain crystal planes, resulting in the inverted pyramids seen in the figure. The most important part of the structure for the subsequent photoelectrochemical etching is the point (apex) of the pyramids. The pitch of the pyramid apexes in the figure is approximately $7 \mu \mathrm{m}$.

Figure 3 shows extremely deep high aspect-ratio holes, nearly $50 \mu \mathrm{m}$ deep, formed in single-crystal silicon by photoelectrochemical etching (Lau et al. $1995 a, b)$. In this technique, n-type silicon forms the anode of an electrochemical etching cell using dilute hydrofluoric acid (HF) as the etchant. This type of etching requires the presence of holes for the dissolution of the silicon, and as we are in n-type material these holes need to be provided by illuminating the silicon with light of energy greater than the band gap. These photogenerated holes migrate through the silicon to the high field region at the pyramid apexes, so dissolution only takes place at these points (not along the walls) if the etch conditions are just right. As an aside, a quasi-three-dimensional PC structure can be formed by modulating the etch current density, which in turn will modulate the width of the photoelectrochemically etched hole (Charlton et al. 1997). If the structure in figure 3 is repetitively thermally oxidized, and the oxide subsequently removed, then eventually a matrix of free-standing single-crystal pillars of silicon will form as shown in figure 4 .

The structures shown in figures 3 and 4 are both interesting forms of PC, each favouring a particular polarization state of light. Band gaps preferentially form for transverse electric (TE) polarized light in a connected lattice as depicted in figure 3, while band gaps preferentially form for transverse magnetic polarized light in a lattice of isolated high index regions as shown in figure 4. The silicon structure shown in figure 4 was the basis of a PC shown to have an incomplete band gap in the near infrared part of the spectrum (Charlton et al. 1997). For all its positive benefits, silicon also has a major disadvantage in trying to fabricate $\mathrm{PC}$ devices. The major disadvantage is that it is opaque to wavelengths less than 


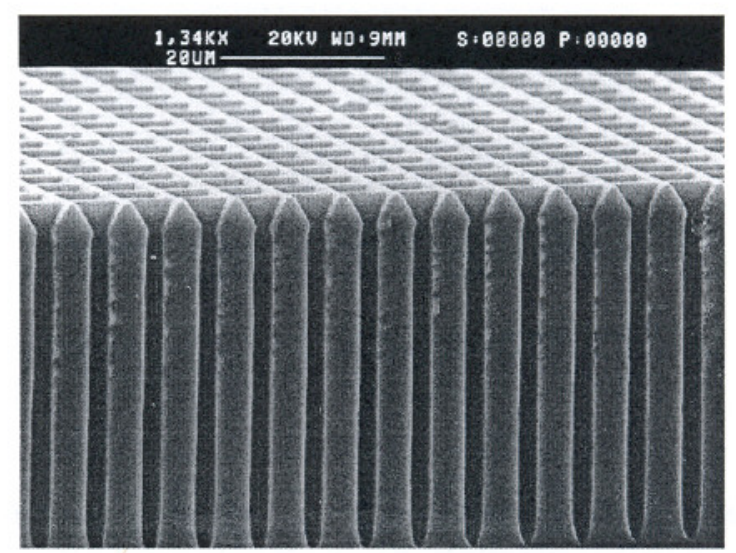

Figure 3. High aspect-ratio holes in silicon.

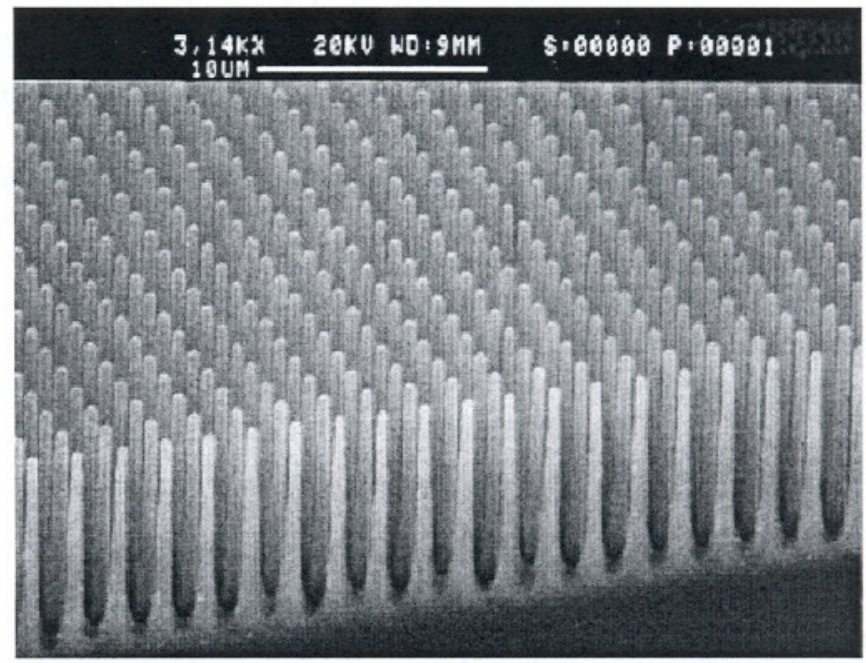

Figure 4. Single-crystal silicon pillars.

about a micrometre, which means that PCs operating in the visible part of the spectrum cannot be based on a macroporous silicon matrix.

As we needed to work in the visible region of the spectrum we had to change both the host matrix material and the waveguide structure. Charlton \& Parker (1997) chose the relatively high index material silicon nitride $(n \sim 2.02)$ for the core of a planar waveguide, with silicon dioxide $(n \sim 1.46)$ as the upper and lower cladding layers. This form of planar waveguide, with the PC formed by etching air holes into the silicon nitride core, formed the basis of virtually all of the visible wavelength $\mathrm{PC}$ work carried out at the University of Southampton. An SEM cross-section of a $\mathrm{PC}$ formed in a silicon nitride membrane structure is shown in figure 5 .

In figure 5, a triangular lattice of air holes has been etched completely through the waveguiding silicon nitride core of a planar waveguide. For the purposes of clarity, the top cladding oxide has been removed using HF, and this has also 


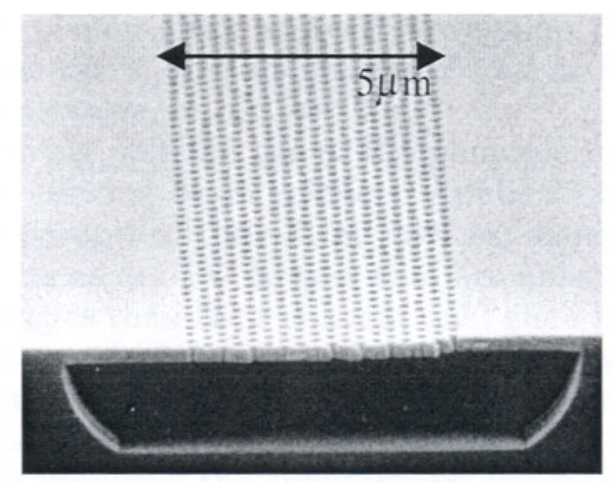

Figure 5. Photonic crystal formed in a silicon nitride membrane.

removed the oxide underlying the central $\mathrm{PC}$ region. The whole structure is formed on a single-crystal silicon wafer substrate. This structure provides a good deep band gap for TE polarized light and can be used as the basis for filters, beam-splitters and dispersion control elements.

Once we have a robust generic design for testing out potential PC devices and circuits, we are in a position to make full use of the rich photonic band structure a PC offers for applications in optical technologies. Just as in the semiconductor band structure case, the slope of the photonic band structure is a measure of the photon group velocity in the $\mathrm{PC}$, this has far-reaching practical implications. Netti et al. (2001) showed that having a photonic band structure at our disposal brings with it some non-intuitive consequences, such as being able to create materials exhibiting multirefringence when simple birefringence is the only possibility in conventional bulk optical materials.

\section{Some applications of photonic crystals}

Clearly, we can engineer the overall shape of the photonic bands, to some extent, by the choice of the optical matrix material and the geometry we choose for the placement of the air holes. As the slope of the photonic bands will give rise to positive or negative dispersion, we are able to achieve dispersion engineering using PC structures, and we have shown that a $1 \mathrm{~mm}$ length of PC can effectively replace over $100 \mathrm{~km}$ of special dispersion compensating optical fibre. Clearly, at the photonic band gap edges, where the slope of the band approaches zero, we can make light come to a grinding halt! This is no more than the case of the electron (or hole) being completely Bragg reflected at the Brillouin zone boundary in a semiconductor crystal, but it clearly shows that by propagating light in a PC close to a band edge we can slow it down to an arbitrarily low speed, opening up the possibility of optical delay lines and optical buffers (optical memory). It is these very powerful potential applications of PC devices and circuits that are generating so much interest in these structures worldwide. The other reason these devices are so interesting is the miniaturization that is possible using this technology. Above we saw the size saving in comparing a planar PC dispersion compensator with its optical fibre equivalent, huge reductions in area are also possible by replacing standard planar glass circuits 
and devices (e.g. a planar glass arrayed waveguide grating), with their PC counterparts. In these cases, area reductions of four to five orders of magnitude seem to be easily achievable.

Applications involving just the use of the photonic band gap include devices such as filters and polarizing beam splitters. Applications utilizing the photonic band structure include optical time-delay devices, dispersion compensators and superprisms. Superprisms are PC devices that are able to refract incoming light over much greater angles than their conventional glass prism counterparts, with clear applications in wavelength division multiplexing systems (Baumberg et al. 2004). If, and when, we are able to produce perfect three-dimensional PC structures, a whole new world is opened up to us. Lodahl et al. (2004) showed that we can now start to influence the spontaneous emission decay rates of photon emitters as originally discussed in the papers by Bykov \& Yablonovitch. A practical outcome of this extreme control over the properties of light would be the fabrication of extremely high efficiency, zero-threshold lasers, clearly a product of enormous commercial interest and the subject of a great deal of current research.

Finally, a well-publicized application of PC waveguiding structures is the formation of waveguides with bend radii of the order of the wavelength of light. Such PC waveguides would be required for integration of optical devices with current-day electronic circuits, since conventional waveguide geometries and structures produce guides with bend radii far too large to be accommodated on a typical electronic circuit. A problem encountered by people working in this area is the loss of light at the waveguide bend, even in the PC case. In the early days of examining this problem it seemed to be a simple matter to fill in a row of air holes in a bulk PC to produce a guided light path for photons, including paths around very tight radius bends. Why this seemed so simple, in the beginning, was that the codes used to model these structures were two-dimensional, so all the light that entered the waveguide bend nicely coupled around the bend to emerge undiminished at the far side. Unfortunately, Nature is not so kind to us, real PC waveguides are three-dimensional structures and it was found, in practice, that a considerable amount of light incident on a sharp waveguide bend coupled out of the top of the crystal into free space and was lost! It is not too difficult to see why this may happen in some cases. Light incident on a tight bend will 'see' the PC boundary at a large range of incident angles, and unless a complete band gap is present (all angles and all polarization states) the light will not be tightly confined to the waveguide. This is clearly very similar to the situation considered right at the beginning of this paper when we were trying to make a $\mathrm{PC}$ with a complete gap, and the solution is, therefore, the same. If you want to make a PC which has similar properties at all incident angles, then it is a good idea to pick a 'crystal' geometry that 'looks' the same over all incident angles. It is possible to create such isotropic (high symmetry) crystal geometries, but they are not normally found in Nature. These structures are generally known as quasicrystals.

\section{Photonic quasicrystals}

In creating planar waveguiding $\mathrm{PCs}$ using direct-write electron beam lithography, we are in the enviable position of being able to place the air holes wherever we like in the plane of the dielectric, and not necessarily in a nice neat

Phil. Trans. R. Soc. A (2006) 

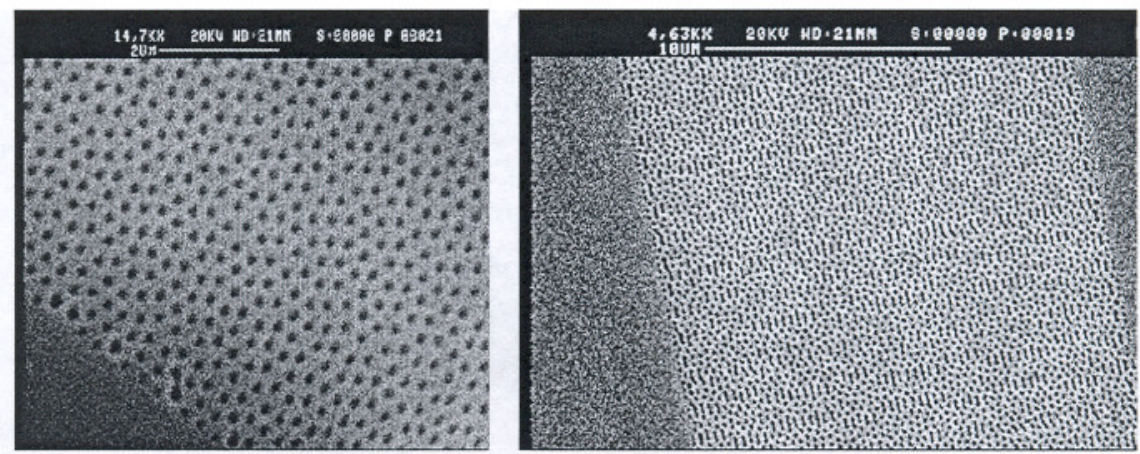

Figure 6. SEM photomicrographs of a 12-fold symmetric waveguiding photonic quasicrystal fabricated in silicon nitride. One micrometre bar marker on the left-hand image and $10 \mu \mathrm{m}$ bar marker on the right. Note how the structure almost looks random, although it is formed from a regular square/triangle tiling procedure.
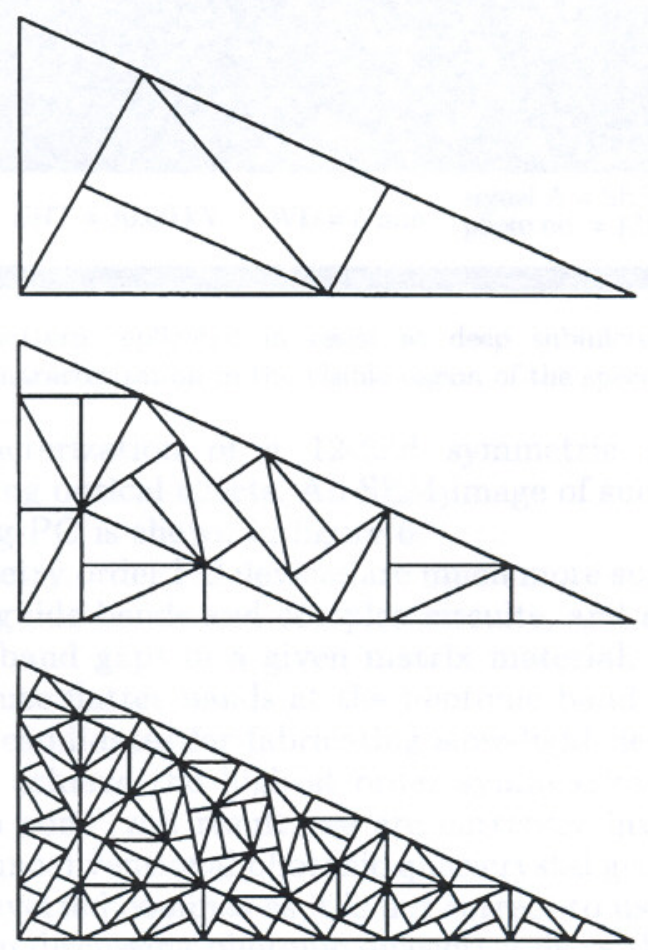

Figure 7. Formation of the 'Pinwheel' structure after Conway.

crystallographic pattern. Quasicrystals are very interesting geometric constructs that allow the possibility of producing symmetries not normally found in Nature, the very well-known fivefold symmetric Penrose tiling being such an example. Higher order symmetries will allow a greater degree of isotropy and fortunately it is possible to create high order symmetries using quasicrystals.

The highest order symmetry in the plane using conventional lattice structures is 6 (triangular lattice). Zoorob et al. (2000) previously reported the fabrication 


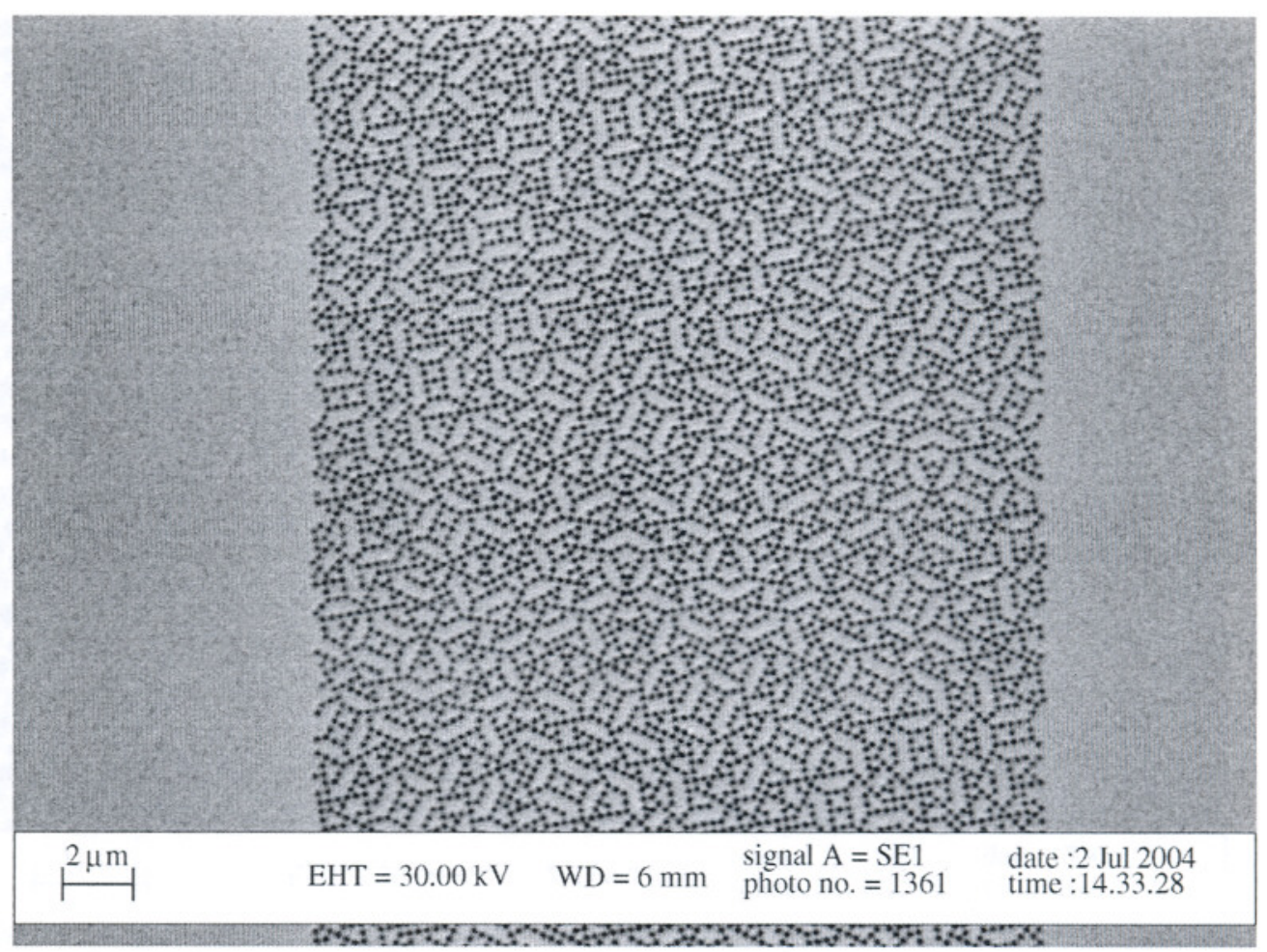

Figure 8. Pinwheel pattern replicated in resist at deep submicron dimensions for optical characterization in the visible region of the spectrum.

and optical characterization of a 12-fold symmetric photonic quasicrystal exhibiting interesting optical effects. An SEM image of such a 12-fold symmetric planar waveguiding $\mathrm{PC}$ is shown in figure 6.

Such high symmetry order PC devices are much more suitable for guiding light around tight waveguide bends and complex circuits, and are also more likely to produce complete band gaps in a given matrix material. High symmetry order PCs will also produce flatter bands at the photonic band edges, and as a result will be favourable candidates for fabricating slow-light devices.

In the quest to achieve the highest order symmetries possible, so that the roundest 'Brillouin zone' will result, we are currently investigating the optical properties of very unconventional photonic quasicrystal geometries. I have placed Brillouin zone in inverted commas as it is not correct to use the terms 'lattice' or Brillouin zone when discussing photonic quasicrystals as these quantities are not strictly defined in these short-range disordered, long-range ordered structures.

The first structure I would like to discuss is the 'Pinwheel' structure first discovered by the mathematician John Conway (although not published!). The routine used to form the Pinwheel is shown diagrammatically in figure 7 .

Conway discovered that it was possible to decompose a $1,2, \sqrt{5}$ right-angled triangle into five congruent triangles. These five triangles could then each be decomposed into five more congruent triangles, and so on. Senechal $(1996 a, b)$ showed that by continuing with this process a structure of infinite symmetry can be formed, the number of orientations is infinite because the smallest angle of the 


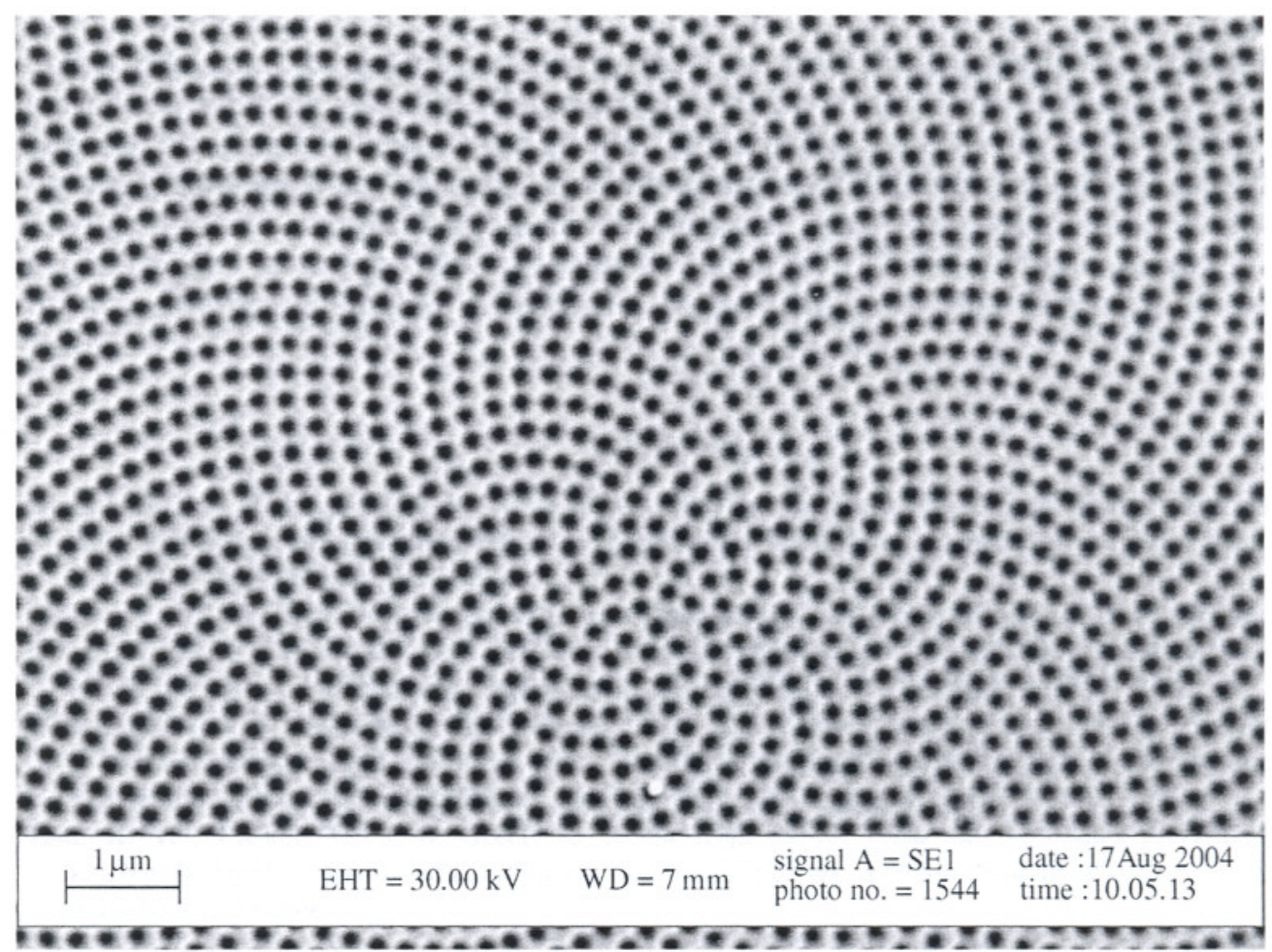

Figure 9. A very high symmetry photonic quasicrystal replicated in resist based on the seed arrangement in a sunflower. The resulting diffraction pattern is circular with no strong underlying lower symmetry orders apparent.

triangle, $\theta=\arctan (1 / 2)$, is irrational with respect to $\pi$. The coordinates of the Pinwheel structure's vertices can be used as the input file for running a directwrite electron beam lithography system, so it is possible to draw and etch this pattern into a silicon nitride waveguide for subsequent optical characterization. Figure 8 shows the Pinwheel pattern replicated in a resist mask on silicon nitride prior to dry etching the air holes into the silicon nitride waveguide core.

Although the infinite symmetry implied by the Pinwheel structure should produce a circular diffraction pattern, in fact there is quite a strong underlying eightfold symmetry visible in the diffraction pattern resulting from this structure (Senechal $1996 a, b$ ).

In the search for higher symmetry in the resulting diffraction pattern we arrived at another photonic quasicrystal structure, this time based on a Sunflower seed arrangement (figure 9). In this case the diffraction pattern that results from modelling the structure is much more isotropic, it is circular with no apparent underlying lower order symmetries (bright spots) apparent, unlike the Pinwheel structure.

\section{Conclusions}

It seems almost perverse that greater functionality can be imparted to a material by taking most of the material away! However, this is seen to be the case with the 
porous dielectric materials known as PCs. In looking at rather esoteric structures, such as photonic quasicrystals, we have barely begun to scratch the surface of what is possible. For example, we have not even started to see the effect of altering the basis of the crystal. If instead of a simple air hole, what if we used multiple air holes, or even multiple air holes of different radius? If instead of having distinct regular lattices or quasicrystal structures, what happens if we grade the PC to merge from one type to the other? There is almost certainly an infinite number of further possibilities to investigate in this very rich research field.

\section{References}

Baumberg, J. J., Perney, N. M. B., Netti, M. C., Charlton, M. D. B., Zoorob, M. E. \& Parker, G. J. 2004 Visible wavelength super-refraction in photonic crystal superprisms. Appl. Phys. Lett. 85, 354-356. (doi:10.1063/1.1772521)

Bykov, V. P. 1972 Spontaneous emission in a periodic structure. Sov. Phys. JETP 35, 269-273.

Charlton, M. D. B. \& Parker, G. J. 1997 Fabrication of high aspect ratio silicon microstructures by anodic etching. J. Micromech. Microeng. 7, 155-158. (doi:10.1088/0960-1317/7/3/020)

Charlton, M. D. B., Roberts, S. W. \& Parker, G. J. 1997 Guided mode analysis, and fabrication of a 2-dimensional visible photonic band structure confined within a planar semiconductor waveguide. Mater. Sci. Eng. B B49, 155-165. (doi:10.1016/S0921-5107(97)00102-5)

Lau, H. W., Parker, G. J. \& Greef, R. 1995 a High aspect ratio silicon pillars fabricated by electrochemical etching and oxidation of macroporous silicon. EMRS Spring Meeting, Strasbourg, France.

Lau, H. W., Parker, G. J., Greef, R. \& Hölling, M. $1995 b$ High aspect ratio submicron pillars fabricated by photoassisted electrochemical etching and oxidation. Appl. Phys. Lett. 67, 1877-1879. (doi:10.1063/1.114362)

Lehmann, V. 1993 The physics of macropore formation in low doped n-type silicon. J. Electrochem. Soc. 140, 2836-2843.

Lodahl, P., van Driel, A. F., Nikolaev, I. S., Irman, A., Overgaag, K., Vanmaekelbergh, D., Voss, W. L. A. F., Nikolaev, I. S., Irman, A. \& Overgaag, K. 2004 Controlling the dynamics of spontaneous emission from quantum dots by photonic crystals. Nature 430, 654-656. (doi:10. 1038/nature02772)

Netti, M. C., Harris, A., Baumberg, J. J., Whittaker, D. M., Charlton, M. D. B., Zoorob, M. E. \& Parker, G. J. 2001 Optical trirefringence in photonic crystal waveguides. Phys. Rev. Lett. 86, 1526-1529. (doi:10.1103/PhysRevLett.86.1526)

Senechal, M. 1996 a Quasicrystals and geometry, p. 220. Cambridge: Cambridge University Press. ISBN 0521575419.

Senechal, M. 1996 b Quasicrystals and geometry, p. 243. Cambridge: Cambridge University Press. ISBN 0521575419 .

Yablonovitch, E. 1987 Inhibited spontaneous emission in solid state physics and electronics. Phys. Rev. Lett. 58, 2059. (doi:10.1103/PhysRevLett.58.2059)

Zoorob, M. E., Charlton, M. D. B., Parker, G. J., Baumberg, J. J. \& Netti, M. C. 2000 Complete photonic bandgaps in 12-fold symmetric quasicrystals. Nature 404, 740-743. (doi:10.1038/ 35008023) 